\title{
Piezoelectricity-induced terahertz photon absorption by confined acoustic phonons in wurtzite CdSe nanocrystals
}

\author{
Tzu-Ming Liu, ${ }^{1}$ Meng-Ju Yang, ${ }^{2}$ Chih-Wei Lai, ${ }^{2}$ Pi-Tai Chou, ${ }^{2}$ Ming-Hao Chang, ${ }^{3}$ Hsiang-Lin Liu, ${ }^{3}$ and Chi-Kuang Sun ${ }^{1,4, *}$ \\ ${ }^{1}$ Department of Electrical Engineering and Graduate Institute of Electro-Optical Engineering, National Taiwan University, \\ Taipei 10617, Taiwan \\ ${ }^{2}$ Department of Chemistry, National Taiwan University, Taipei 10617, Taiwan \\ ${ }^{3}$ Department of Physics, National Taiwan Normal University, Taipei 11650, Taiwan \\ ${ }^{4}$ Research Center for Applied Sciences, Academia Sinica, Taipei 11529, Taiwan
}

(Received 22 November 2007; published 26 February 2008)

\begin{abstract}
We report here the observation of piezoelectricity-induced terahertz photon absorption related to confined acoustic phonons in nanoparticles. Terahertz photon inactive $(l=0)$ breathing modes become terahertz photon active in wurtzite CdSe nanocrystals due to the piezoelectric coupling. Obvious absorption peaks related to $(l=0)$ modes appeared in the terahertz absorption spectra and the frequency of the peaks were inversely proportional to the size of the CdSe nanocrystals. This piezoelectrically coupled electric vibration provides a mechanism for low-dimensional systems to convert a terahertz photon into a phonon of the same frequency.

DOI: 10.1103/PhysRevB.77.085428 PACS number(s): 77.65. $-\mathrm{j}$, 61.46.Hk, 63.22. $-\mathrm{m}$, 78.30. $-\mathrm{j}$
\end{abstract}

\section{INTRODUCTION}

The piezoelectric effect, the coupling of electric fields and stress fields in matter, is well known in bulk materials such as CdSe. In low-dimensional systems, the piezoelectricity of nanowires has been exploited to demonstrate nanoforce sensors $^{1}$ and nanogenerators. ${ }^{2}$ In quantum dots, the piezoelectric coupling can change their electronic and optical properties. ${ }^{3}$ However, such classically piezoelectric couplings happened in a steady state in which the generated electric field adiabatically followed the applied force. There is still no evidence in low-dimensional systems for dynamic piezoelectric coupling between acoustic vibrations and electromagnetic waves. In low-dimensional nanostructures, due to spatial confinement, propagating acoustic waves form three dimensional standing waves and show energy quantization. The corresponding eigenfrequencies of thus confined acoustic phonons fall in the terahertz regime and can be tailored by the size of the particles. ${ }^{4-6}$ Therefore, if the nanocrystals have intrinsic piezoelectricity, without the help of foreign charges, ${ }^{6}$ the confined acoustic vibrations should fluctuate the dipole moment of nanocrystals and couple with the incident terahertz photons.

In this paper, we report the observation of piezoelectricity-induced terahertz photon absorption related to the confined acoustic phonons in nanoparticles. By using a Fourier transform infrared (FTIR) spectroscopy system we measured the terahertz absorption spectra of wurtzite (piezoelectric) CdSe nanocrystals. The terahertz photon inactive $(l=0)$ breathing modes were found to become terahertz photon active in wurtzite CdSe nanocrystals due to the piezoelectric coupling. The frequency of the measured resonant absorption was inversely proportional to the size of the $\mathrm{CdSe}$ nanocrystals. The corresponding terahertz photon absorption cross section has approximately quadratic dependence on the particle size, which agrees well with a theoretical model based on elastic continuum theory. This piezoelectric coupled electric vibration provides a mechanism for lowdimensional systems to convert a terahertz photon into a phonon of the same frequency.

\section{SAMPLE PREPARATION AND EXPERIMENT}

The vibrational modes of a free homogeneous sphere are classified by the torsional and the spheroidal (SPH) modes. ${ }^{7}$ They can be further indexed by $l$ and $n,{ }^{7}$ where $l \geqslant 0$ is the angular momentum and $n \geqslant 0$ is a mode index. Idealizing the nanocrystals as small isotropic spheres, symmetry arguments ${ }^{8}$ show that only SPH modes can be observed by photon interactions. Low frequency Raman inelastic light scattering sees only $l=0$ and $l=2$ modes, ${ }^{4}$ pump-probe pulsed laser experiments see only $l=0$ modes, ${ }^{5}$ and the foreign-charge assisted terahertz photon absorption sees only $l=1$ modes. ${ }^{6}$ Since the above-mentioned symmetry argument assumes that the material is isotropic, this rules out piezoelectric coupling, which might induce terahertz photon absorption related to the confined acoustic phonons other than (SPH, $l=1)$ modes. To investigate this, we prepared wurtzite (piezoelectric) CdSe nanocrystals for FTIR spectroscopy system to measure their terahertz photon absorption spectra.

Preparation of the wurtzite CdSe nanocrystals from a $\mathrm{CdO}$ green-chemistry precursor was performed according to a method previously reported by Chen et al. ${ }^{9}$ Briefly, a stock solution containing selenium powders was prepared by dissolving Se powders in tri- $n$-butylphosphine, and then these were diluted with di- $n$-octylamine. A mixture of $\mathrm{CdO}$ and stearic acid was gently heated in a three-neck flask to $140{ }^{\circ} \mathrm{C}$ under Ar flow. After the $\mathrm{CdO}$ reaction was complete, the mixture was allowed to cool to room temperature. Then, tri$n$-octylphosphine oxide (TOPO) and hexadecylamine were added to the flask, and the mixture was heated to $320^{\circ} \mathrm{C}$. At this temperature, the Se injection solution was quickly injected into the hot solution. The reaction mixture was allowed to cool to $290{ }^{\circ} \mathrm{C}$ for the growth of the CdSe nanocrystals. The sizes of CdSe nanocrystals could be controlled by terminating the reaction at different times of cooling. The CdSe nanocrystals were then precipitated out from the growth solution by adding methanol. To study the size dependence of terahertz absorption, we synthesized five batches of CdSe nanocrystals with different sizes. Measuring 

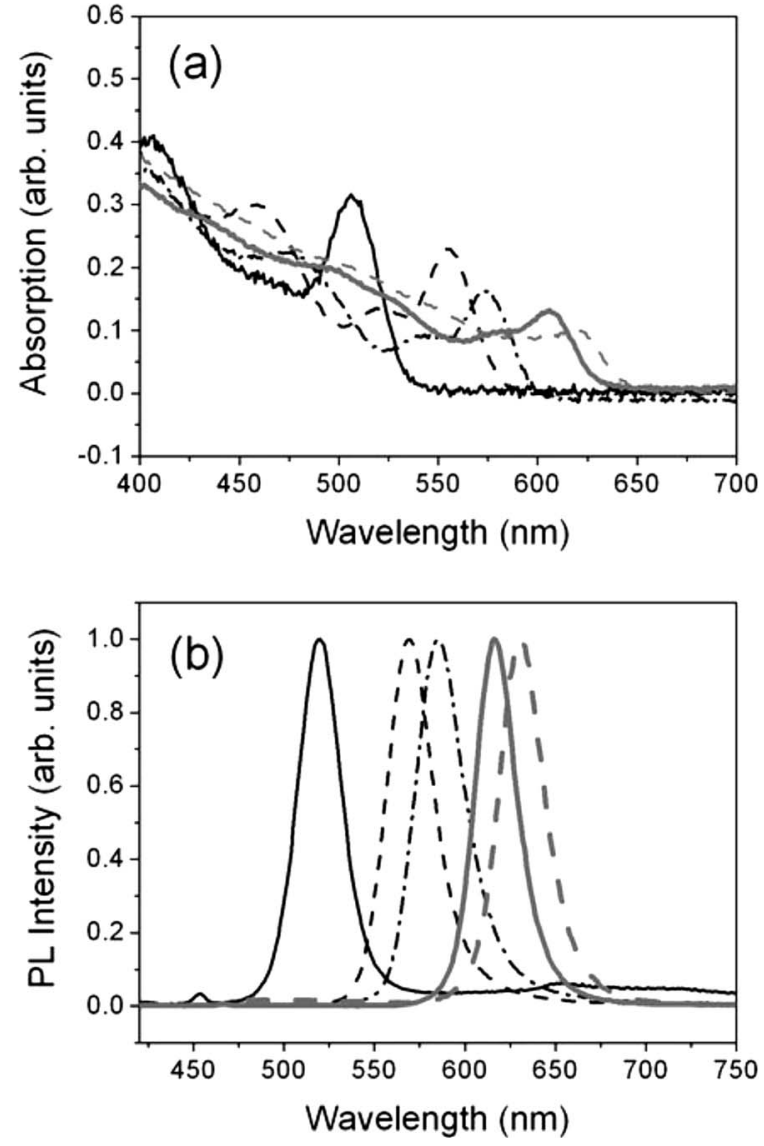

FIG. 1. Room temperature (a) optical absorption and (b) photoluminescent spectra of CdSe nanocrystals with $3.4 \mathrm{~nm}$ (solid black), $4.1 \mathrm{~nm}$ (dashed black), $4.4 \mathrm{~nm}$ (dash-dotted black), $5.6 \mathrm{~nm}$ (solid gray), and $5.8 \mathrm{~nm}$ (dashed gray) particle diameters.

with a transmission electron microscope, their mean diameters $D$ were found to be $3.4,4.1,4.4,5.6$, and $5.8 \mathrm{~nm}$, respectively, with $10 \%-20 \%$ size variations. To characterize how spherical the particles are, we arbitrarily chose 50 particles from the transmission electron microscope image with nanometer resolution and measured the equatorial and polar radius of the $\mathrm{CdSe}$ nanoparticles. The statistical ratio of the short axis over the long axis is 0.91 , which is very close to that of a sphere. With a strong confinement of the electronic wave function $\left(D<2 a_{B} \sim 10 \mathrm{~nm}\right.$, where $a_{B}$ is the exciton Bohr radius of $\mathrm{CdSe}$ ) and a uniform size distribution, we observed clear excitonic absorption [Fig. 1(a)] and sharp $(\delta \lambda \sim 30 \mathrm{~nm})$ photoluminescence peaks [Fig. 1(b)] of the $\mathrm{CdSe}$ nanocrystals at room temperature. As the particle size decreased, both the excitonic absorption and the photoluminescence peaks blueshifted to shorter wavelengths (Fig. 1). These results confirm the quality of our samples and are consistent with the trend of size dependency. ${ }^{10}$ In order to avoid the terahertz photon absorption from solutions, we used centrifugation and obtained the powders under nitrogen flow. All of the nanocrystals were wrapped by a passivation layer of TOPO (mass density $\rho_{m}=880 \mathrm{~kg} / \mathrm{m}^{3}$, longitudinal sound speeds $V_{L m}=2407 \mathrm{~m} / \mathrm{s}$, and transverse sound speeds $\left.V_{T m}=1203 \mathrm{~m} / \mathrm{s}\right),{ }^{11}$ which is softer than CdSe $(\rho$ $=5810 \mathrm{~kg} / \mathrm{m}^{3}$, spherically averaged longitudinal sound speeds $V_{L}=3570 \mathrm{~m} / \mathrm{s}$, and spherically averaged transverse sound speeds $\left.V_{T}=1720 \mathrm{~m} / \mathrm{s}\right) .{ }^{12}$ It can be treated as an acoustically isotropic matrix of the CdSe nanocrystals. ${ }^{11} \mathrm{~A}$ dimensionless actual number of CdSe nanocrystals within the interrogated volume from 3.4 to $5.8 \mathrm{~nm}$ nanocrystals were $3.7 \times 10^{17}, 1.6 \times 10^{17}, 7.6 \times 10^{16}, 4.4 \times 10^{16}$, and $4.5 \times 10^{16}$, respectively. These nanocrystal powders were separately sealed by two polyethylene films (30 $\mu \mathrm{m}$ thick) for the convenience of holding in terahertz photoabsorption measurements. The overall sample thickness was $\sim 500 \mu \mathrm{m}$.

\section{RESULTS}

The terahertz photoabsorption measurements were made by a Bruker IFS 66v Fourier transform infrared spectrometer. For the reduction of water vapor absorption, all the samples were held in a vacuum chamber with 3-4 mbar air pressure. The CdSe samples were illuminated by a terahertz beam with $\sim 3 \mathrm{~mm}$ diameter. The transmitted FTIR signals were recorded by a liquid-helium-cooled silicon bolometer. We first measured the transmitted power spectrum of the polyethylene film alone as the background reference. Then, we measured the terahertz photoabsorption spectra of the CdSe nanocrystals with polyethylene films at room temperature. The inset of Fig. 2(a) shows a typical FTIR trace of the $5.8 \mathrm{~nm}$ sample. Around the frequency of $17.8 \mathrm{~cm}^{-1}$, there is an excess absorption dip (indicated by a black arrow) over the background electric-dipole absorption. ${ }^{13}$ Then, we calculated the corresponding spectrum of the total extinction cross section by

$$
\sigma_{e x}=\frac{\ln (T) A}{N},
$$

where $T$ is the transmission, $A$ is the excitation area, and $N$ is the number of nanocrystals in the excitation area [see Fig. 2(a)]. By removing the electric-dipole absorption induced background, we can obtain the spectrum of extinction-crosssection related to the excess absorption (ECEA) [dashed gray trace in Fig. 2(b)]. At the maximum of extinction, the ECEA of each $5.8 \mathrm{~nm}$ CdSe nanocrystal is found to be $6 \times 10^{-22} \mathrm{~m}^{2}$. With similar measurements and analysis, we also obtained the spectra of ECEA for other sizes of CdSe nanocrystals [Fig. 2(b)]. They all show obvious absorption peaks (indicated by arrows) and the frequency of peak absorption blueshifts as the particle size decreases. To check if the resonant terahertz absorption originates from the confined acoustic modes, we first calculate the mode frequency $\omega_{0}$ from the eigenfrequency equation for the $(\mathrm{SPH}, l=0)$ modes, ${ }^{7}$

$$
\tan \left(\xi_{0}\right)\left(1-\eta_{0}^{2} / 4\right)=\xi_{0},
$$

where $\xi_{0}=\omega_{0} D /\left(2 V_{L}\right)$ and $\eta_{0}=\omega_{0} D /\left(2 V_{T}\right)$. Since TOPO is much softer than $\mathrm{CdSe}$, the real part of the eigenfrequency modified by the presence of the TOPO matrix was much smaller than our experimental error. We thus treat the eigenfrequency as $\omega_{0}$ in our following analysis. Another issue is the linewidth broadening. It might result from either homogeneous broadening or inhomogeneous broadening. Homo- 

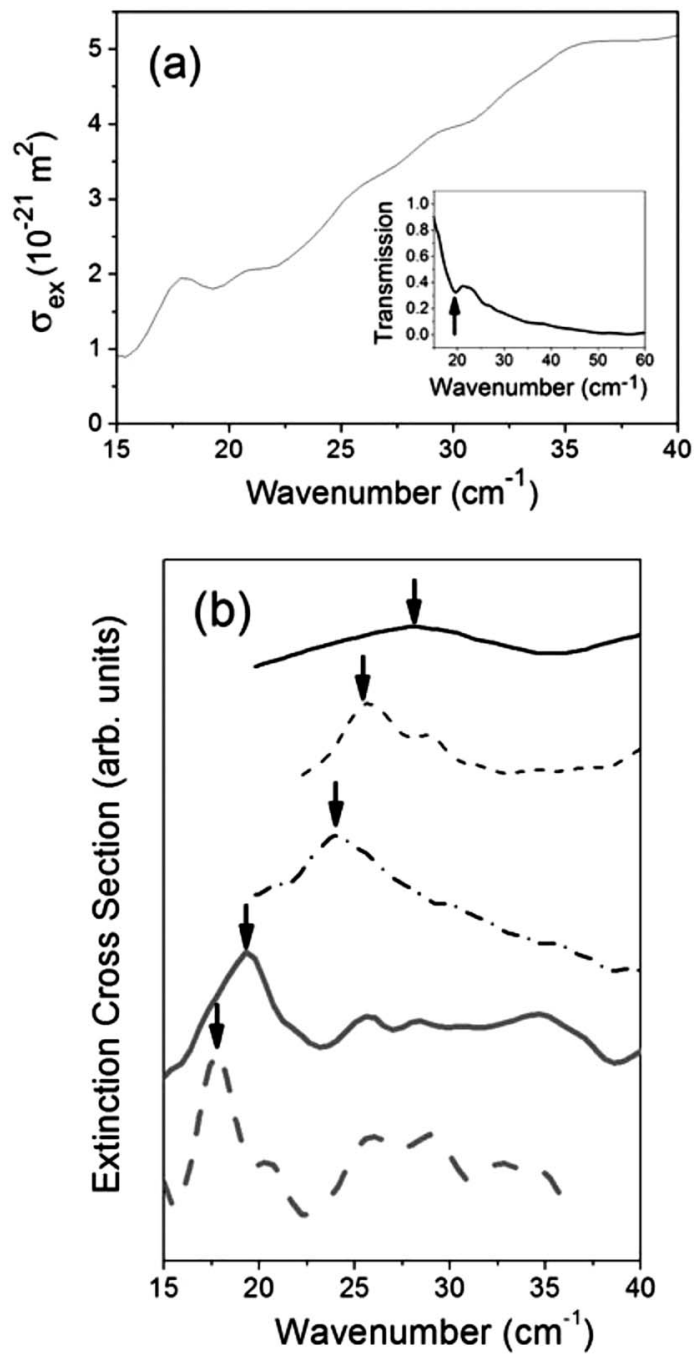

FIG. 2. (a) Extinction-cross-section spectrum of excess terahertz photon absorption of $5.8 \mathrm{~nm}$ CdSe nanocrystals, calculated from the transmission spectra (inset figure) measured by a Fourier transform infrared (FTIR) spectroscopy system. (b) The extinction-crosssection spectra of excess terahertz absorption of CdSe nanocrystals corresponding to the samples characterized in Fig. 1.

geneous broadening might come from either finite acsoutic quality factor $Q$ or elastic anisotropy. Using the material parameters of CdSe (Ref. 12) and TOPO,${ }^{11}$ we calculated the acoustic impedance contrast for $(\mathrm{SPH}, l=0)$ modes. ${ }^{14}$ The resulting $Q$ is around 17.7. To evaluate the contribution from elastic anisotropy, we took the extreme values of the $V_{L}(3505-3793 \mathrm{~m} / \mathrm{s})$ and the $V_{T}$ $(1506-1840 \mathrm{~m} / \mathrm{s})$ (Ref. 15) into Eq. (2). The average variation of eigenfrequency $\omega_{0}$ is about $8 \%$. These results suggest that the broadening of terahertz absorption linewidth in our measurement should be dominated by the inhomogeneous size distribution, rather than the homogeneous acoustic contrast or elastic anisotropy. Taking the acoustic parameters of CdSe (Ref. 11) into Eq. (2), we calculate and plot the frequencies of the $(\mathrm{SPH}, l=0)$ fundamental mode as a function of the inverse particle diameter in Fig. 3 (solid line). Considering the linewidth broadening effects, good agreement with the experimentally measured terahertz absorption peak posi-

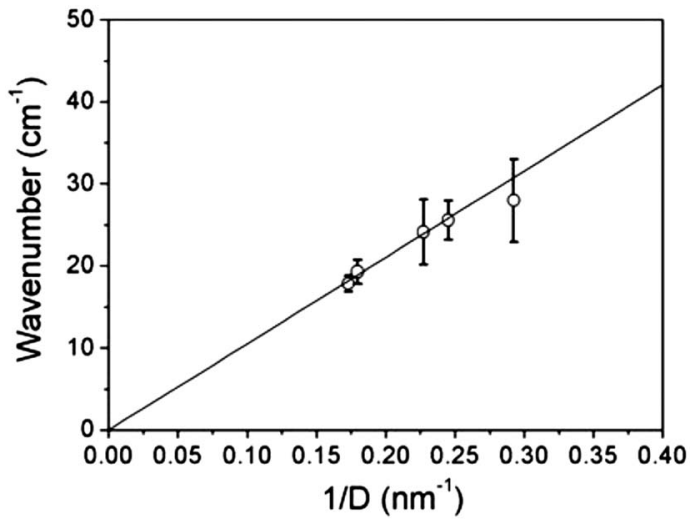

FIG. 3. Frequencies of the measured resonant terahertz photon absorption peak versus inverse particle diameters of CdSe nanocrystals (open circles). Error bars represent the bandwidth of the resonant absorption. The data points agree well with the calculated eigenfrequency as a function of inverse particle diameter (solid line).

tion (open circles) can be found. This result indicates that the excess terahertz resonant absorption we observed in $\mathrm{CdSe}$ nanocrystals is related to the $(\mathrm{SPH}, l=0)$ mode, which was originally considered forbidden in nonpiezoelectric materials.

\section{THEORETICAL MODEL}

To explore the role that piezoelectric coupling plays in the processes of $(\mathrm{SPH}, l=0)$ related terahertz photon absorption and to estimate the size dependence of this coupling, we developed a theoretical model, where we idealize the CdSe nanocrystals as homogeneous piezoelectric elastic continuum spheres with mass density $\rho$ and radius $R$. Subjected to an isotropic strain $s$ of $(\mathrm{SPH}, l=0)$ breathing mode, the induced polarization is $P_{i}=e_{i j k} s_{j k}$, where $e_{i j k}$ is the piezoelectric constant tensor and $s_{j k}$ is the strain. For the wurtzite $(6 \mathrm{~mm})$ symmetry, nonzero components of $e_{i j k}$ are $e_{z x x}, e_{z y y}, e_{z z z}, e_{y y z}$, and $e_{x x z}{ }^{16}$ They result in $P_{x}=P_{y}=0$ and $P_{z}=\left(2 e_{z x x}+e_{z z z}\right) s$ $=\epsilon_{0}\left(\epsilon_{z z}-1\right) E_{z}$ where $\varepsilon_{z z}$ is the relative dielectric constants of $\mathrm{CdSe}$ for the electric field along the $z$ axis. Therefore, the fundamental breathing mode is an excitation which can induce dipole moment change along the $z$ axis of wurtzite $\mathrm{CdSe}$. The terahertz photons could thus couple with the breathing mode at its natural resonant frequency $\omega_{0}$. Since the particle size $(3-5 \mathrm{~nm})$ is much less than the wavelength of terahertz electromagnetic waves, we can consider the nanocrystals to be in a quasi-cw electric field. The nanocrystals will thus expand with an increase of radius: $\Delta r=s R$ $=g E R$, where $g$ is the coupling factor between the strain and the local electric field $E$. In addition, the local electric field $E$ in the nanocrystals should be linked to the global electric field $E_{g}$ with a local field factor $f$ by $E=3 E_{g} \epsilon_{m} /\left(\epsilon+2 \epsilon_{m}\right)$ $=f E_{g}$, where $\varepsilon$ and $\varepsilon_{m}$ are dielectric constants of CdSe and the matrix, respectively. For time dependent analysis, we followed the treatment used in the Ref. 5. Since the fundamental breathing mode has a radial symmetry, we simplified the formulation by a one dimensional simple harmonic oscillator 
with force constant $k_{s p}$. The mass $m$ of the nanocrystals can be linked to $k_{s p}$ by $m \omega_{0}^{2}=k_{s p}$. We also include a damping coefficient $b$ to consider the mechanical coupling of the nanocrystals to the matrix. In the static case, the externally applied force on the simple harmonic oscillator is $F_{\text {ext }}$ $=k_{s p} \Delta r$. Consequently, $F_{\text {ext }}=k_{s p} f E_{g} R g$, which directly relates the applied electric field $E_{g}$ to the force driving the simple harmonic oscillator. With this driving force, the equation of motion of the simple harmonic oscillator becomes

$$
m \frac{d^{2} r}{d t^{2}}=F_{e x t}-b \frac{d r}{d t}-k_{s p} r
$$

Using phasor analysis, we obtain $\left(k_{s p}+i \omega b-m \omega^{2}\right) A=F_{\text {ext }}$ where $A$ is the amplitude of $r$ and $\omega$ is the frequency of the applied electric field. When $F_{\text {ext }}=0$, the free vibrations are damped and their frequency $\omega$ is complex valued with a positive imaginary part $\gamma$ which is equal to $b /(2 m)$, and a real part $\omega_{1}$. The quality factor of the mode $Q$ is $\omega / \Delta \omega$, where $\gamma$ is equal to $\Delta \omega / 2$ and thus $Q$ is equal to $\omega_{1} /(2 \gamma)$. Consequently, the ratio of $b$ over $m$ will be equal to that of $\omega_{1}$ over $Q$. At resonance, the time-averaged power absorption by this driven damped simple harmonic oscillator is $P_{a v}=\left(k_{s p} f E_{g} R d_{33}\right)^{2} /(2 b) .{ }^{5}$ By taking into account the random orientation of the nanoparticles, the effective average value of $E^{2}$ is reduced to one-third. Accordingly, consider only light damping $\left(\omega_{0} \approx \omega_{1}=\operatorname{Re}(\eta) V_{T} / R\right)$, the $P_{a v}$ averaged over the ensemble is $\overline{P_{a v}}=2 \pi \rho Q \operatorname{Re}(\eta)^{3} V_{T}^{3} f^{2} E_{g}^{2} R^{2} g^{2} / 9$. Dividing $\overline{P_{a v}}$ by the incident energy flux $s=c \epsilon_{0} E_{g}^{2} / 2$, where $c$ is the speed of light and $\epsilon_{0}$ is the permittivity in vacuum, the ECEA can be expressed as

$$
\sigma_{E C E A}=\frac{4 \pi \rho Q \operatorname{Re}(\eta)^{3} V_{T}^{3} f^{2} R^{2} g^{2}}{9 c \varepsilon_{0}} .
$$

As described above, soft TOPO will not result in a large imaginary part of the eigenfrequency and thus $\operatorname{Re}(\eta)$ is very close to $\eta_{0}$, which is equal to 5.764 . The local field factor $f$ is calculated to be 0.54 by the dielectric constant of $\mathrm{CdSe}$ $(\epsilon=8.82)$ (Ref. 17) and TOPO $\left(\epsilon_{m}=2.5\right) .{ }^{18}$ For a free sphere, the coupling factor $g$ between the strain and the applied electric field cannot be larger than that determined by the classical relation of $c_{i j k l} s_{k l}=e_{i j k} E_{k}$, where $c_{i j k l}$ is the stiffness constants. The corresponding $g$ is equal to the element of $e_{i j k}$ devided by $c_{i j k l}$, which is on the order of $4 \times 10^{-12} \mathrm{C} / \mathrm{N}$ for CdSe. ${ }^{15}$ Inserting all these numerical factors and material parameters of CdSe (Ref. 11) into Eq. (4), $\sigma_{E C E A}$ are found to be around $10^{-21} \mathrm{~m}^{2}$, which are on the same order with our measured results (Fig. 4, solid squares). From the $\log 10-\log 10$ plot of $\sigma_{E C E A}$ (Fig. 4) versus the particle diam-

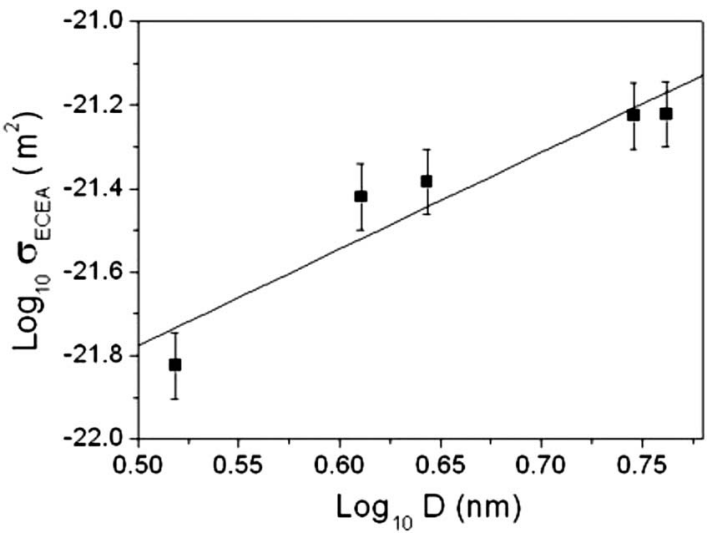

FIG. 4. Log 10- $\log 10$ plot of the measured extinction cross section of excess terahertz photon absorption versus the particle diameters of CdSe nanocrystals (solid squares). The fitting line (solid curve) has a slope of 2.3.

eters, the data fitting line has a slope of 2.3, which is close to our theoretical prediction that $\sigma_{E C E A}$ is proportional to $R^{2}$. These results support our hypothesis that the piezoelectric coupling would induce excess terahertz resonant absorption with the breathing modes of the CdSe nanocrystals.

\section{CONCLUSION}

In conclusion, we observed the piezoelectric coupled terahertz resonant photon absorption related to the confined acoustic modes in nanoparticles. By measuring the FTIR spectra of CdSe nanocrystals with different sizes, we showed that the resonant frequency of terahertz photon absorption is inversely proportional to the particle size. These frequencies are in agreement with the (SPH, $l=0)$ acoustic breathing modes predicted by the elastic continuum theory. Using a simple electromechanical model, we explain how electric fields drive the vibration of the acoustic breathing modes through piezoelectric coupling. The measured extinction cross sections also support the predicted $R^{2}$ dependence. Our result suggests a mechanism for low-dimensional systems to convert a terahertz photon into a phonon of the same frequency.

\section{ACKNOWLEDGMENTS}

This project is sponsored by the National Science Council of Taiwan under Grant Nos. of NSC96-2120-M-002-014 and NSC 96-2628-E-002-043-MY3 and by the National Taiwan University Research Center for Medical Excellence. We thank D. B. Murray and L. Saviot for technical discussions on the dipolar modes in a nanosphere.
*Corresponding author.

${ }^{1}$ X. Wang, J. Zhou, J. Song, J. Liu, N. Xu, and Z. L. Wang, Nano Lett. 6, 2768 (2006).

${ }^{2}$ X. Wang, J. Song, J. Liu, and Z. L. Wang, Science 316, 102
(2007).

${ }^{3}$ C. Cornet, A. Schliwa, J. Even, F. Doré, C. Celebi, A. Létoublon, E. Macé, C. Paranthoën, A. Simon, P. M. Koenraad, N. Bertru, D. Bimberg, and S. Loualiche, Phys. Rev. B 74, 035312 (2006). 
${ }^{4}$ E. Duval, A. Boukenter, and B. Champagnon, Phys. Rev. Lett. 56, 2052 (1986).

${ }^{5}$ T. D. Krauss and F. W. Wise, Phys. Rev. Lett. 79, 5102 (1997).

${ }^{6}$ D. B. Murray, C. H. Netting, L. Saviot, C. Pighini, N. Millot, D. Aymes, and H.-L. Liu, J. Nanoelectron. Optoelectron. 1, 92 (2006).

${ }^{7}$ H. Lamb, Proc. London Math. Soc. 13, 189 (1882).

${ }^{8}$ E. Duval, Phys. Rev. B 46, 5795 (1992).

${ }^{9}$ C.-Y. Chen, C.-T. Cheng, C.-W. Lai, Y.-H. Hu, P.-T. Chou, Y.-H. Chou, and H.-T. Chiu, Small 1, 1215 (2005).

${ }^{10}$ C. J. Murphy, Anal. Chem. 74, 520A (2002).

${ }^{11}$ S. Banerjee, S. Jia, D. I. Kim, R. D. Robinson, J. W. Kysar, J.
Bevk, and I. P. Herman, Nano Lett. 6, 175 (2006).

${ }^{12}$ D. L. Rode, Phys. Rev. B 2, 4036 (1970).

${ }^{13}$ S. I. Lee, Tae Won Noh, K. Cummings, and J. R. Gaines, Phys. Rev. Lett. 55, 1626 (1985).

${ }^{14}$ L. Saviot and D. B. Murray, Phys. Rev. Lett. 93, 055506 (2004).

${ }^{15}$ V. V. Zubritskiǔ, Tech. Phys. 42, 639 (1997).

${ }^{16}$ D. Royer and E. Dieulesaint, Elastic Waves in Solids I (SpringerVerlag, Berlin 2000), Chap. 3.

${ }^{17}$ M. C. Beard, G. M. Turner, and C. A. Schmuttenmaer, Nano Lett. 2, 983 (2002).

${ }^{18}$ J. Jiang, T. D. Krauss, and L. E. Brus, J. Phys. Chem. B 104, 11936 (2000). 